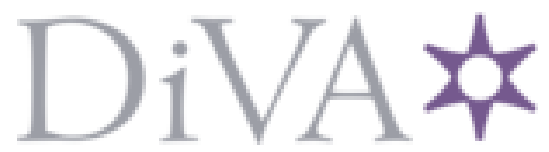

http://www.diva-portal.org

This is the published version of a paper published in Electric power systems research.

Citation for the original published paper (version of record):

Jürgensen, J H., Nordström, L., Hilber, P. (2016)

Individual failure rates for transformers within a population based on diagnostic measures.

Electric power systems research, 141: 354-362

http://dx.doi.org/10.1016/j.epsr.2016.08.015

Access to the published version may require subscription.

N.B. When citing this work, cite the original published paper.

Permanent link to this version:

http://urn.kb.se/resolve?urn=urn:nbn:se:kth:diva-192290 


\title{
Individual failure rates for transformers within a population based on diagnostic measures
}

\author{
Jan Henning Jürgensen*, Lars Nordström, Patrik Hilber \\ KTH Royal Institute of Technology, School of Electrical Engineering, Osquldas väg 10, 10044 Stockholm, Sweden
}

\section{A R T I C L E I N F O}

\section{Article history:}

Received 2 December 2015

Received in revised form 29 June 2016

Accepted 11 August 2016

\section{Keywords:}

Asset management

Condition monitoring

Diagnostic measures

Failure rate

Failure rate modeling

Health index

\begin{abstract}
A B S T R A C T
The high monetary value of a transformer has placed the transformer life-time optimization into the focus of asset management. The average failure rate has created reasonable results within reliability modeling, however, it cannot reflect the probability of failure for an individual transformer. In this paper, a method is introduced to calculate individual failure rates for a transformer population based on failure statistics and diagnostic measurements such as dissolved gas, and 2-furfuraldehyde analysis. The method is applicable to all types of components and the comprehensibility makes it effective for practical implementation. The results are evaluated against two health indices based on a weight factor and fuzzy logic. It can be observed that the presented individual failure rates are plausible representatives of the transformer's probability of failure. Therefore, the results can also be utilized for asset management decision-making.
\end{abstract}

(c) 2016 Elsevier B.V. All rights reserved.

\section{Introduction}

Continuous improvement of the power system to achieve secure electricity supply is a major goal of energy utilities. Minimizing costs through optimized investments and maintenance of equipment support the ambition to remain competitive in a challenging market. The power transformer is the component with the highest individual monetary value and its operation is significant to a reliable grid. Consequently, the assessment of its actual condition is critical in the work towards an optimized transformer life time by scheduling maintenance and replacement.

Condition monitoring $(\mathrm{CM})$ is a technique which combines data acquisition, data processing and maintenance decision-making [1]. This technique found wide acceptance in production plants by the late 1990s [2]. In 2002, a review for condition assessment of power transformer was conducted in [3] that concluded an increasing need for diagnostic and monitoring tools. The further development of diagnostic measurements such as dissolved gas analysis (DGA), frequency response analysis (FRA), etc. has led to several publications, summarized in [4] in a bibliography survey. Furthermore, [5] discusses CM techniques as one of the three major transformer asset management activities and highlights the latest research.

\footnotetext{
* Corresponding author.

E-mail addresses: jan-henning.jurgensen@ee.kth.se (J.H. Jürgensen), larsn@ics.kth.se (L. Nordström), hilber@kth.se (P. Hilber).
}

\subsection{Health indices}

One tool to effectively use and combine all condition-based diagnostics into a single value is the health index [6]. It illustrates the overall condition of a single transformer and supports asset management decisions. The health index is utilized to estimate the end of life, as an indication of risk of failure or to plan maintenance strategies [7].

There are several approaches to calculate the health index in research as well as in practice. The main approach is the allocation of a weight factor to the diagnostic tests to calculate the health index [8-11]. After a score is assigned to the transformer, the result is graded into the categories: very good, good, moderate, bad, and very bad. These linguistic terms are simplified expressions of the transformer condition. In this approach, numerical values define the threshold between the linguistic categories of the diagnostic measurements. However, to set a clear threshold of a diagnostic measure to determine the condition is difficult because in practice there are overlaps of scores. To address this challenge, fuzzy methods have been applied to the calculation of the health index as an alternative approach [12-15]. Other approaches are Bayesian networks [16] or artificial neural networks [17].

The advantages of the health index are better decision-making, identifying end of life assets, allocation of risk, and planning maintenance strategies. One challenge is the determination of the weight factors for the diagnostic tests which requires experience of transformer experts. Moreover, the application of fuzzy methods or neural networks as techniques to calculate a health index 
requires knowledge about the techniques themselves. Especially, the complexity of fuzzy logic increases if more measurements are added. More input data results in more rules in the fuzzy-logic model which increases computation time as well as the need for more precise expert knowledge for its definition.

\subsection{Failure rate modeling}

One probabilistic reliability measure is the failure rate. The failure rate is defined as

$$
\begin{aligned}
\lambda(t) & =\lim _{\Delta t \rightarrow 0} \frac{P(t<T \leq t+\Delta t \mid T>t)}{\Delta t} \\
& =\lim _{\Delta t \rightarrow 0} \frac{F(t+\Delta t)-F(t)}{\Delta t} * \frac{1}{R(t)}=\frac{f(t)}{R(t)}
\end{aligned}
$$

where $F(t)$ is cumulative distribution function, $f(t)$ the probability density function, and $R(t)$ is the reliability function. Thus, the failure rate is the probability that a component fails within the next time interval $(t, \Delta t]$. The failure rate is constant if an exponential lifetime distribution is assumed for the population. Due to the long lifetimes of power system components, the statistical failure rate estimation might be difficult and an average failure rate is the result of experience or observed data [18,19].

In asset management, the failure rate can be used to model component reliability and thus has an impact on network reliability modeling and optimal maintenance planning [20]. Hence, it is critical that models which can link maintenance activities to component failure rates exist. For example, the failure rate has been used to link maintenance and reliability to develop a reliabilitycentered asset maintenance method [21]. This method has been applied to 20-kV XLPE service aged cables [22], or to wind power plants $[23,24]$. The failure rate is also an input parameter in riskbased maintenance optimization [25] or to demonstrate the effect of imperfect preventive maintenance [26]. In addition, the failure rate is a crucial parameter in power system reliability assessment to calculate component availabilities, reliability block diagrams, Markov models, and interruption indices $[27,28]$.

So far, the average failure rate $\lambda$ is allocated to all components in a population [29]. Although applying the average failure rate to all components of a population is practical, it cannot characterize the actual condition of each individual component. Every component is unique and the external environment such as weather, location in the grid, usage, and maintenance influence the wear process differently. As a result, developed models might underestimate or overrate component reliability for certain time periods [29]. [30] describes the limits of a constant failure rate for system reliability models and suggests that actual failure distributions of components must be determined as accurately as possible. Another drawback with average failure rates is that the impacts of maintenance activities are difficult to identify. Therefore, efforts to increase reliability are hardly determinable.

The proposed method is based on the approach to estimate failure rates from condition information. Modeling the failure rate by using equipment inspection data has been presented in [29]. The method in [29] reflects component criticality in system models and allows a more precise distribution of customer reliability. Failure rate estimation as part of an integrated general asset management model for electric utilities has been presented in [31]. The model in [31] is based on a Hidden Markov model which is a modification of [32]. To determine the condition of transformers, transition probabilities are calculated between states that represent predefined transformer conditions. In [33], a method is introduced to calculate condition based failure rates for electrical network equipment with failure statistics on subcomponent level and a weight factor. These methods for condition-based failure rate estimation are discussed and applied in [34].

The proposed method, however, utilizes the idea of using equipment inspection data to calculate a component specific failure rate but, in contrast to the previously mentioned methods, it does not require the definition of the deterioration process or a condition score. Moreover, [29] describes the challenge of determining the failure rate of the best and worst inspection outcome which is necessary for the interpolation. This determination is not required for the proposed method in this paper. Compared to the aforementioned methods, the proposed method also does not require a large amount of failure and condition monitoring data to estimate an individual failure rate for a component. [29] identified the limited amount of input data as a general problem in the power system domain. This illustrates the need for a method which is able to calculate results even with a small amount of input data and without the definition of condition states. The advantage of the proposed method is that these challenges are overcome with the idea of relating the condition of components to each other. This enables the estimation of the individual probability of failure as a relative risk.

\subsection{Article content and structure}

In this paper, we propose a time independent method based on reliability measures, failure statistics and information from diagnostic measurements to estimate the probability of failure of transformers by deriving individual failure rates from the average failure rate of the population. The method could be applied as a decision-making tool for maintenance and therefore makes it comparable to a health index. The benefit of the suggested method is the comprehensibility. It is easy to understand and to apply to all types of components. Another benefit is the use of the results in ensuing system reliability calculations. The results are useful for conducting reliability analysis in a system context with tools such as block diagrams or fault trees.

The next section presents and explains the details of the method. Section 3 shows how the method is applied to a transformer population of 30 transformers with data provided from [12]. Afterwards, the results are discussed and compared to health indices and an asset management tool. The last two sections conclude the work and describe the future development.

\section{Method}

The foundation of the proposed method was presented in [35]. Here, FRA was applied to a set of transformers to identify transformers with a higher probability of failure due to winding distortions. The authors show the failure rate as a function of transformers with a detected deviation and conclude that these have a higher failure rate if just a small number of transformers with winding distortions were detected. In conclusion, the results indicate that adding condition-based information to the average failure rate of a population shows which components have an increased probability of failure, so that further analysis can be conducted. In this section, we develop and formalize the methods previously discussed.

\subsection{Characterization of the average failure rate with failure statistics}

Assume a population of components named $N$ in its useful life period with an average failure rate $\lambda_{N}$. It is assumed that the average failure rate is the result of failures that happen over a specific time interval. Every failure is the consequence of one particular failure cause and occurs in a specific failure location. The variable $i=1$, 
$\ldots, n$ describes all defined failure locations which are associated with the population $N$ and are gathered from historical failure data. These are statistically analyzed and the proportion of the failure locations is defined as vector $\alpha_{i}$. The failure location is the part of the component where the failure has occurred. The average failure rate can be expressed as

$\lambda_{N}=\lambda_{N} \sum_{i=1}^{n} \alpha_{i}$

where $n$ is the number of predefined failure locations and $\sum_{i=1}^{n} \alpha_{i}=$ 1 . Now, the average failure rate is described as the sum of causespecific failure rates which is the competing risk approach [36]. The identification of a cause-specific failure rate is necessary to relate a condition measurement to the correct failure location. For example, the water content in the transformer oil indicates an accelerating of the ageing of the paper insulation and thus can be related to winding failures but has no significance as a condition indicator for failures of the cooling unit. However, this approach assumes an independence of failures.

The failure location data can be obtained during a failure mode and effect analysis (FMEA) which is for example discussed in [27]. One part of the FMEA is the identification of all failure modes and failure causes. One approach to identify failure modes, failure causes, and failure locations is the failure analysis of historical population data. For example, [37] investigates a transformer population in terms of failure locations, failure modes, failure origins, actions, and external effects. The choice of which failure statistics to use, depends on the available measurement and to what extend it is able to indicate the failures. In this paper, failure location data is used to identify in which subcomponent of the transformer the failure has occurred. Consequently, failure locations are associated with certain parts of a transformer such as windings, on-load tap-changer, core, tank, and so forth. If failure locations cannot be determined they must be represented as unknown, to achieve a sum of 1 . The failure definition should be clear while defining failure locations or using failure statistics. For instance, a failure can be defined as an outage of the equipment or a possible failure which has been repaired during a planned outage. Failure location data is used because it can indicate subsystems which fail more frequently. This awareness can help to monitor specific subsystems as well as to focus on the correct maintenance tasks.

The extension of the average failure rate with vector $\sum_{i=1}^{n} \alpha_{i}=$ 1 demonstrates that by adding population properties to $\lambda_{N}$, the reliability measure can be expressed more detailed without a change. In fact, the average failure rate is the result of historical failures occurred in a specific time interval in a population. These component failures can have different causes and might occur in different locations among the population and are a indication of critical subcomponents. The intention is to specify the characteristics of the average failure rate to identify the element which can be modified by component information to calculate an individual failure rate (IFR). The next section continues to characterize $\lambda_{N}$.

\subsection{Considering the uncertainty of measurements}

The proposed method utilizes diagnostic measurements that have been developed to gather information to indicate certain component failures. However, [38] states in clause 3.2 that measurements are not exact. In [39], clause 3.1.2, it is further stated that "the result of a measurement is only an approximation or estimate of the value of the measurand" which suggests that an ideal result exists [40]. This ideal result of a measurement is known as target value $x$. Furthermore, the basic post-measurement model is defined as

$\theta=x+e, \quad e \sim N\left(0, \sigma^{2}\right)$

where $e$ is the measurement error following the normal distribution with mean 0 and variance $\sigma^{2}$ and $\theta$ is the measured value. Assuming all components of error are evaluated and corrections are applied to the measurement, a doubt remains that the result of the measurement represents the correct target value. This doubt is known as the concept of uncertainty and therefore describes in a general sense the validity of the result of a measurement [39]. Now, we determine the probability $\rho_{j}$ which describes the assurance that the diagnostic measure $j$, corresponding to the failure location $i=j$, is successful. To do so, the concept of the 'confidence interval' (CI) is used here which is discussed in more detail in [40]. According to [40], the CI can be defined as "a valid 95\% confidence interval for $x$ is a random interval that has probability at least $95 \%$ of covering $x$ no matter what the values of all unknown parameters". A measurement process that creates this interval is considered as successful and generally expressed it can be stated that we are $95 \%$ sure that the measurement process was successful [40]. Thus, $\rho_{j}=95 \%$ would describe that there is a $95 \%$ assurance that the measurement process is successful. Hence, the average failure rate can be further characterized as

$\lambda_{N}=\lambda_{N} \sum_{j=i=1}^{n}\left(\rho_{j} \alpha_{i}+\left(1-\rho_{j}\right) \alpha_{i}\right)$

with $\sum_{j=i=1}^{n}\left(\rho_{j} \alpha_{i}+\left(1-\rho_{j}\right) \alpha_{i}\right)=1$ for $i=1, \ldots, n$. The term $\rho_{j} \alpha_{i}$ illustrates the probability that the created $\mathrm{CI}$ of diagnostic measurement $j$ contains the target value, while $\left(1-\rho_{j}\right) \alpha_{i}$ states the probability that the target value lies not in the $\mathrm{CI}$ and is not successful.

The assurance $\rho_{j}$ is also known as confidence level and is depending on the person responsible for the analysis how to set it. It is recommended that the confidence level should be taken from an independent international standard organization which has developed a technical standard for the measurement of interest. Now, the confidence interval can be determined by

$\bar{\theta}+z_{\rho_{j} / 2} \frac{\sigma}{\sqrt{n}} \leq \mu \leq \bar{\theta}+z_{\rho_{j} / 2} \frac{\sigma}{\sqrt{n}}$

where $\bar{\theta}$ is the sample mean, $\sigma$ the sample standard deviation, $z_{\rho_{j} / 2}$ the $N(0,1)$ value of $\rho_{j}$ assuming the normal distribution, $n$ the number of samples, and $\mu$ is the real mean which is the value of interest for $\theta_{j, k}$. However, the use of an interval is not practical for calculations in this method and the sample mean $\bar{\theta}$ serves as the value of interest for further calculations.

\subsection{Introduction of a diagnostic measurement factor}

In Eq. (4), the term $\rho_{j} \alpha_{i}$ was identified as the probability that a diagnostic measure successfully creates a $\mathrm{CI}$ that contains the target value and therefore it is seen as the part that includes information to indicate a future failure. The next step is the utilization of information from measurement $j$. Therefore, we introduce the diagnostic measurement factor $S_{j, k}$ that describes the trend of the obtained diagnostic measure $\theta_{j, k}$ gathered from $j$ of component $k$. The factor $S_{j, k}$ represents the deviation of $\theta_{j, k}$ from the average diagnostic measure $\theta_{j, N}$. The average diagnostic measure is given by

$\theta_{j, N}=\frac{1}{m} \sum_{k=1}^{m} \theta_{j, k}$ 
with $m$ as the size of population $N . \theta_{j, N}$ is the average value of all obtained values $\theta_{j, k}$ of measurement $j$. Since measurement $j$ reflects a failure indication for failure location $i$, it can be associated with the term $\rho_{j} \alpha_{i}$. Assume that $\theta_{j, k}$ deviates from the average and thereby indicates the individual condition of $k$. Then, $S_{j, k}$ can be used to adjust $\lambda_{N}$ to calculate an individual failure rate $\lambda_{\text {IFR, } k}$. Hence, we compute

$\lambda_{\text {IFR }, k}=\lambda_{N} \sum_{j=i=1}^{n}\left(S_{j, k} \rho_{j} \alpha_{i}+\left(1-\rho_{j}\right) \alpha_{i}\right)$

$S_{j, k}$ is a function of $\theta_{j, k}$ and depending on the diagnostic measure the behavior varies. Thus, the function space is expected to be large. To limit the intricacy of the proposed method, the method adopts the assumption of a linear relationship between measurements and condition according to $[8,11]$. Therefore, two functional types are proposed for the calculation of the diagnostic measurement factor $S_{j, k}$ : a positive linear and a negative linear. Fig. 1 depicts how the behavior of $S_{j, k}$ could vary with the suggested two functions depending on $\theta_{j, k}$. These functions describe how $\theta_{j, k}$ manipulates $\lambda_{N}$ to gain an individual representation $\lambda_{\text {IFR,k }}$ of the condition of $k$. The positive linear function is defined as

$S_{j, k}= \begin{cases}0 & \text { if } \theta_{j, k}<0 \\ \frac{1}{\theta_{j, N}} \theta_{j, k} & \text { if } \theta_{j, k} \geq 0\end{cases}$

an the negative linear function is characterized as

$S_{j, k}= \begin{cases}\frac{\theta_{p c, j}-\theta_{j, k}}{\theta_{p c, j}-\theta_{j, N}} & \text { if } \theta_{j, k} \leq \theta_{p c, j} \\ 0 & \text { if } \theta_{j, k}>\theta_{p c, j}\end{cases}$

with $\theta_{p c, j}$ as the value that describes the perfect condition of a component. The perfect condition is here defined as a "as new" condition.

The definition of the two functions does not consider a critical limit of the obtained measures. A critical limit $\theta_{c l, j}$ could be defined as the value, when the obtained measure clearly indicates a failure and replacement of the component is required. The determination of the critical values is difficult because in-depth knowledge of the diagnostic measures is mandatory to specifically estimate a value to describe the component as failed or not working. The IEEE

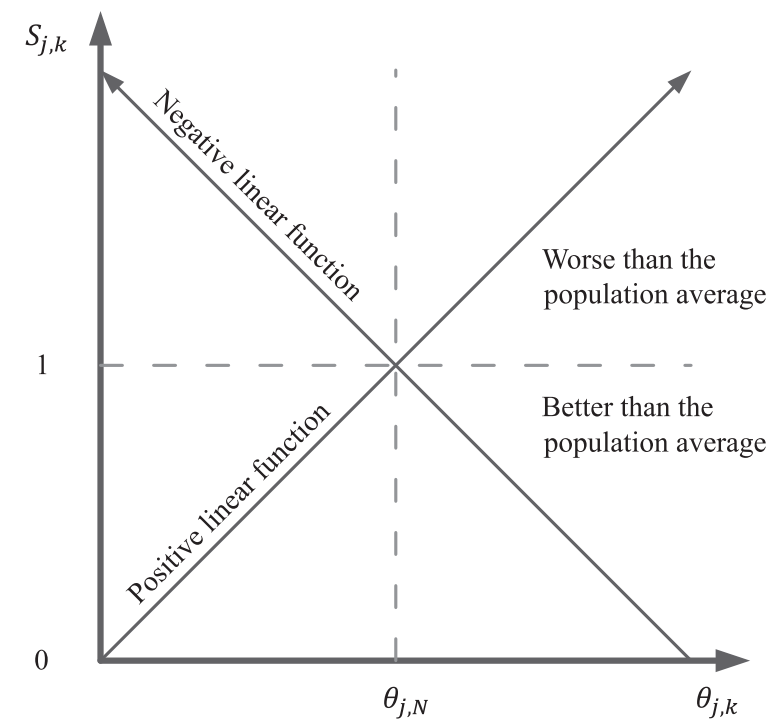

Fig. 1. Schematic representation of the diagnostic measure factor $S_{j, k}$ with respect to the measured value $\theta_{j, k}$. guidelines [41,42] recommend critical limits for certain measurements. However, these limits describe that component property requirements are not fulfilled and that the component is in a bad condition but not necessarily state that a failure has occurred.

\subsection{Selection of the diagnostic measurement factor}

The selection of the type of function can be based on experience, empirical data, investigating literature or expert knowledge. For example, if $j$ measures the water content in transformer oil and it is known that a higher water content is an accelerator of aging [43], selecting a positive increasing function is logical. In contrast, when a measured value decreases and thus impacts the condition of $k$ negatively, we can represent it with a negative linear function.

\subsection{Multiple measurements related to one failure location}

It was assumed that one measurement relates to one failure location, therefore $j=i$. When several measurements provide valid information about one failure location, a weighting of the information is necessary. Now, measurement $j$ related to failure location $i$ is assumed to be a function of the set of measurements $p$. The weights for the set $p$ with $n$ measurements are defined as vector $\mathbf{w}_{j}=\left(w_{j_{1}}, \ldots, w_{j_{n}}\right)^{T}$ with the property $\mathbf{w}_{j}^{T} \mathbf{1}=1$. The elements of $\mathbf{w}_{j}$ are calculated as

$w_{j_{p}}=\frac{c_{p}}{\sum_{i=1}^{n} c_{i}}, \quad p=1, \ldots, n$

where $c_{p} \in[0,10]$ is the weight score assigned to measurement $j_{p}$. A score of $c_{p}=0$ describes no relevance to failure location $i$ and $c_{p}=10$ is interpreted as high relevance of measurement $j_{p}$ to failure location $i$.

The weights describe the significance of each diagnostic measurement to indicate the condition. Thus, the ideal case would be the regression of the diagnostic measurement results towards known inspection outcomes. However, this is often not feasible due to the limited data available [29]. The weights should be chosen based on the relative importance to the overall failure location (subcomponent) condition. These weights can be determined through a collaborative decision-making process based on the opinion of equipment designers and field service personal [29].

\section{Results}

The method presented in the previous section has been applied to a Canadian transformer population with 30 units which are on a voltage level less than $25 \mathrm{kV}$ [12]. Table 1 presents the transformer data, measured values, health indices, and calculated results of the IFR for each transformer. The computation of the IFRs also requires $\lambda_{N}$, failure statistics, weights for the measurements, and the probability $\rho_{j}$ that the measurement process was successful. These data are population properties and hence must be gathered from the population under investigation. However, if the necessary population properties are not available as in this case study, other populations with similar properties must be consulted. The proposed method is shown in a graphical form in Fig. 3 and will be further described with the given case study in this section.

The average failure rate was set to $\lambda_{N}=0.02$, in accordance with [44] which has found wide acceptance in the literature. The transformer failure statistics used are related to the transformer components depicted in Fig. 2. These were presented in [37] based on transformer failure statistics from Germany, Austria, Switzerland, and the Netherlands. Failures in the statistics are defined according to the definitions from CIGRE WG A2.37 and in comparison to other statistics [44-48], it is consistent. A general 
Table 1

Transformer population data with obtained diagnostic measures from 2006, health indices [12] and computed IFR

\begin{tabular}{|c|c|c|c|c|c|c|c|c|c|c|c|c|}
\hline \multirow{2}{*}{$\begin{array}{l}\text { Number } \\
\text { transformer }\end{array}$} & \multirow{2}{*}{$\begin{array}{l}\text { Year of } \\
\text { installation }\end{array}$} & \multirow{2}{*}{$\begin{array}{l}\text { Voltage ratio }(\mathrm{kV}) \text {, and } \\
\text { Power rating }(\mathrm{kVA})\end{array}$} & \multicolumn{6}{|c|}{ Obtained diagnostic measures } & \multicolumn{2}{|l|}{ Health index } & \multirow[t]{2}{*}{$S_{1, k}$} & \multirow[t]{2}{*}{ IFR } \\
\hline & & & $\begin{array}{l}\text { Water } \\
{[\mathrm{ppm}]}\end{array}$ & $\begin{array}{l}\text { Acidity } \\
{[\mathrm{mgKOH} / \mathrm{g}]}\end{array}$ & $\begin{array}{l}\text { BDV } \\
{[\mathrm{kV}]}\end{array}$ & DF [\%] & $\begin{array}{l}\text { DCG } \\
{[\mathrm{ppm}]}\end{array}$ & 2-Fur & $\begin{array}{l}\text { A Weighting } \\
\text { method }\end{array}$ & $\begin{array}{l}\text { B Fuzzy } \\
\text { method }\end{array}$ & & \\
\hline 1 & 1980 & $22 / 6.9 ; 5000$ & 21.7 & 0.024 & 32.5 & 0.075 & 483 & 0.86 & Good & Good & 1.91 & 0.0256 \\
\hline 2 & 1980 & $22 / 6.6 ; 3.15$ & 26.9 & 0.098 & 40.5 & 0.894 & 254 & 0.65 & Good & Good & 1.37 & 0.0223 \\
\hline 3 & 1980 & $22 / 6.6 ; 8$ & 14.5 & 0.033 & 58 & 0.14 & 78 & 0.26 & Good & Good & 0.46 & 0.0167 \\
\hline 4 & 1980 & $22 / 6.9 ; 8$ & 21.2 & 0.226 & 48.7 & 0.424 & 215 & 5.53 & Bad & Bad & 2.55 & 0.0294 \\
\hline 5 & 2000 & $22 / 6.6 ; 5000$ & 10 & 0.01 & 75 & 0.111 & 126 & 0.06 & Good & Very good & 0.48 & 0.0168 \\
\hline 6 & 1981 & $22 / 6.6 ; 4$ & 15.5 & 0.075 & 71 & 0.143 & 38 & 0.53 & Good & Good & 0.40 & 0.0164 \\
\hline 7 & 1981 & $22 / 6.6 ; 10$ & 16.8 & 0.167 & 70.1 & 0.255 & 149 & 0.78 & Good & Good & 0.87 & 0.0192 \\
\hline 8 & 1980 & $22 / 7.2 ; 15$ & 15 & 0.092 & 67.8 & 0.211 & 28 & 0.69 & Good & Good & 0.44 & 0.0166 \\
\hline 9 & 1980 & $22 / 6.9 ; 8000$ & 17 & 0.035 & 62.7 & 0.113 & 9 & 0.21 & Good & Very good & 0.23 & 0.0153 \\
\hline 10 & 1981 & $22 / 3.3 ; 3.15$ & 30 & 0.088 & 37.6 & 0.353 & 197 & 0.31 & Good & Good & 1.00 & 0.0200 \\
\hline 11 & 1970 & $22 / 6.9 ; 8000$ & 16.2 & 0.181 & 25.5 & 0.201 & 35 & 8.76 & Bad & Very bad & 2.89 & 0.0316 \\
\hline 12 & 1980 & $23.5 / 11 ; 25600$ & 15 & 0.155 & 37.5 & 0.182 & 53 & 7.29 & Bad & Very bad & 2.49 & 0.0291 \\
\hline 13 & 1980 & $11 / 23.5 ; 25600$ & 16.8 & 0.115 & 25.6 & 0.174 & 78 & 9.6 & Bad & Very bad & 3.25 & 0.0337 \\
\hline 14 & 1980 & $11 / 23.5 ; 25600$ & 15 & 0.21 & 57.2 & 0.22 & 53 & 6.69 & Bad & Bad & 2.30 & 0.0279 \\
\hline 15 & 1980 & $22 / 6.6 ; 8$ & 27.6 & 0.089 & 30.4 & 0.128 & 336 & 5.12 & Bad & Bad & 2.75 & 0.0307 \\
\hline 16 & 1980 & $21.45 / 6.976 ; 5000$ & 23.5 & 0.106 & 45.8 & 0.207 & 30 & 0.24 & Good & Good & 0.40 & 0.0164 \\
\hline 17 & 1980 & $21.45 / 6.9 ; 8000$ & 24.8 & 0.012 & 29.9 & 0.068 & 504 & 1.68 & Moderate & Moderate & 2.23 & 0.0275 \\
\hline 18 & 1980 & $21.45 / 7.2 ; 15000$ & 23.6 & 0.07 & 39.2 & 0.203 & 22 & 0.5 & Good & Good & 0.46 & 0.0167 \\
\hline 19 & 1980 & $21.45 / 6.9 ; 8000$ & 30.5 & 0.073 & 28.7 & 67 & 30 & 15.7 & Bad & Very bad & 20.23 & 0.1373 \\
\hline 20 & 1980 & $21.45 / 6.9 ; 8000$ & 18.4 & 0.063 & 64.5 & 0.243 & 69 & 0.15 & Good & Good & 0.43 & 0.0165 \\
\hline 21 & 1980 & $22 / 0.4 ; 1600$ & 21.1 & 0.019 & 28.4 & 0.025 & 144 & 0.02 & Moderate & Very good & 0.64 & 0.0178 \\
\hline 22 & 1981 & $22 / 0.4 ; 630$ & 8.1 & 0.01 & 66.9 & 0.042 & 71 & 0.05 & Good & Very good & 0.30 & 0.0157 \\
\hline 23 & 1980 & $23.5 / 11 ; 25600$ & 19.6 & 0.216 & 41.1 & 0.264 & 48 & 7.54 & Bad & Very bad & 2.59 & 0.0297 \\
\hline 24 & 2000 & $22 / 6.98 ; 5000$ & 6 & 0.01 & 67.6 & 0.126 & 427 & 0.08 & Good & Good & 1.39 & 0.0224 \\
\hline 25 & 1981 & $22 / 6.6 ; 10$ & 18.4 & 0.152 & 37.2 & 0.299 & 81 & 1.14 & Moderate & Moderate & 0.84 & 0.0190 \\
\hline 26 & 1997 & $6.6 / 0.4 ; 1600$ & 11.1 & 0.032 & 67.2 & 0.089 & 119 & 0.04 & Good & Very good & 0.47 & 0.0168 \\
\hline 27 & 1980 & $22 / 6.6 ; 3.15$ & 21.5 & 0.147 & 60.8 & 0.938 & 168 & 0.92 & Good & Moderate & 1.15 & 0.0209 \\
\hline 28 & 1986 & $22 / 6.6 ; 4$ & 7.5 & 0.16 & 70.1 & 0.448 & 10 & 0.06 & Good & Good & 0.24 & 0.0154 \\
\hline 29 & 1981 & $0.38 / 19.2 ; 100$ & 13 & 0.091 & 51.6 & 0.369 & 8 & 0.32 & Good & Good & 0.34 & 0.0160 \\
\hline \multirow[t]{4}{*}{30} & 1981 & $0.38 / 19.2 ; 100$ & 35.7 & 0.229 & 41.4 & 0.639 & 24 & 1.07 & Moderate & Moderate & 0.82 & 0.0189 \\
\hline & & Average & 18.15 & 0.081 & 49.35 & 0.26 & 129.57 & 1.36 & & & & \\
\hline & & Critical limits & 35 & 0.2 & 23 & 1.0 & 1920 & 7.3 & & & & \\
\hline & & Perfect values & & & 80 & & & & & & & \\
\hline
\end{tabular}

overview and discussion of transformer statistics can be found in [48].

Table 2 presents the diagnostic measurement methods that were utilized in [12] which correspond to the IEEE guidelines $[41,42]$. Table 2 also shows that the methods are in accordance with the American Society for Testing and Materials (ASTM) standards. The diagnostic measurements are indicating the condition of the liquid and solid insulation of the windings. Hence, all diagnostic measures are associated with winding failures in Fig. 2.

The assumption $j=i$ states that one measurement is related to one specific failure location. For this population, all 6 diagnostic measures are associated with winding failures $i=1$. Therefore, a weighting of the measurements is necessary to relate all 6 diagnostic measures to winding failures. In this case, we assigned a score to each measurement based on $[8,11]$ except that 2-Furfuraldehyde is weighted higher based on the findings in [49]. The weights are shown in Table 2. Corresponding to [8,11], all oil quality measurements combined have a weight of 6 . Thus, the weight 6 was divided over the 4 available measurements. The individual weights also given in $[8,11]$. For example, the water content weight is calculated with $(4 / 11) * 6=2.2$. The 6 diagnostic measurement factors are defined as elements of the vector $\boldsymbol{S}_{j_{p}, k}$. For example, the diagnostic measurement factor $S_{j, k}=\mathbf{w}_{j}^{T} \mathbf{S}_{j, k}$ for transformer number 1 is calculated as follows

$$
\begin{aligned}
S_{1,1}= & \underbrace{0.08}_{w_{1}} * \underbrace{\frac{21.7}{18.15}}_{S_{1_{1}, 1}}+\underbrace{0.02}_{w_{1_{2}}} * \underbrace{\frac{0.024}{0.081}}_{S_{1_{2}, 1}}+\underbrace{0.06}_{w_{1_{3}}} * \underbrace{\frac{80-32.5}{80-49.35}}_{S_{1_{3}, 1}} \\
& +\underbrace{0.06}_{w_{1_{4}}} * \underbrace{\frac{0.075}{0.26}}_{S_{1_{4}, 1}}+\underbrace{0.39}_{w_{1_{5}}} * \underbrace{\frac{483}{129.57}}_{S_{1_{5}, 1}}+\underbrace{0.39}_{w_{1_{6}}} * \underbrace{\frac{0.86}{1.36}}_{S_{1_{6}, 1}}=1.91 .
\end{aligned}
$$

The ASTM used for all measurements a confidence level of 95\% except for measurement 3 , the diagnostic measure of breakdown voltage, where also a $95 \%$ probability of a successful measurement process is assumed here. Likewise the diagnostic measurement factor, the same weights are used for the calculation of $\rho_{1}=95 \%$. Now,

Table 2

\begin{tabular}{|c|c|c|c|c|c|c|c|}
\hline Measurement & Diagnostic measure $j_{p}$ & ASTM Standard & Indicator & $\begin{array}{l}\text { Corresponding to failure } \\
\text { location } i=j \text { in Fig. } 2\end{array}$ & $\begin{array}{l}\text { Confidence } \\
\text { level* }^{*}\end{array}$ & $\begin{array}{l}\text { Assigned score } \\
c_{p}[8,11]\end{array}$ & Weights $w_{j p}$ \\
\hline 1 & Water content & D1533-12 [50] & Oil quality & Windings & $95 \%$ & 2.2 & 0.08 \\
\hline 2 & Acidity & D664-11a [51] & $\begin{array}{l}\text { Overall insulation } \\
\text { system }\end{array}$ & Windings & $95 \%$ & 0.56 & 0.02 \\
\hline 3 & Oil breakdown voltage (BDV) & D1816-12 [52] & Oil quality & Windings & No & 1.64 & 0.06 \\
\hline 4 & Dissipation factor (DF) & D924-08 [53] & Oil quality & Windings & $95 \%$ & 1.64 & 0.06 \\
\hline 5 & $\begin{array}{l}\text { Dissolved combustible gases } \\
\text { (DCG) }\end{array}$ & D3612-02 [54] & Solid insulation & Windings & $95 \%$ & 10 & 0.39 \\
\hline 6 & 2-Furfuraldehyde & D5837-12 [55] & Solid insulation & Windings & $95 \%$ & 10 & 0.39 \\
\hline
\end{tabular}

Used diagnostic measures related to failure locations with absolute reliability. 


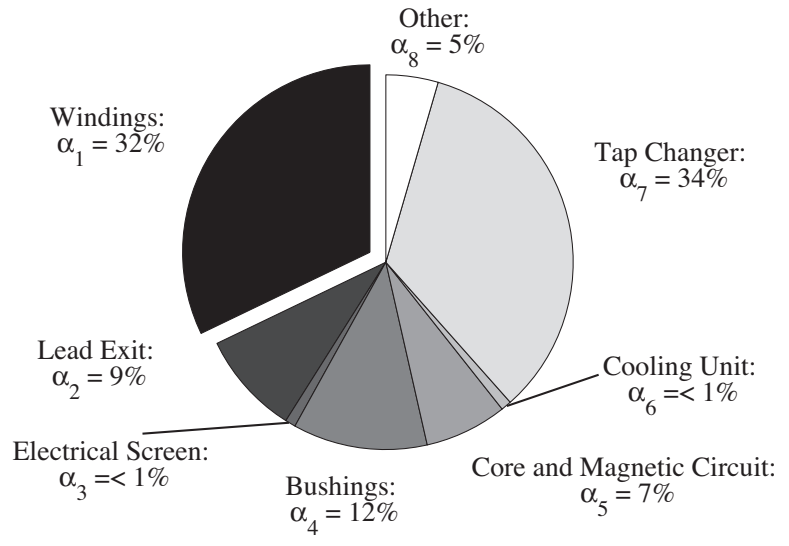

Fig. 2. Transformer failure statistics based on failure location analysis [37].

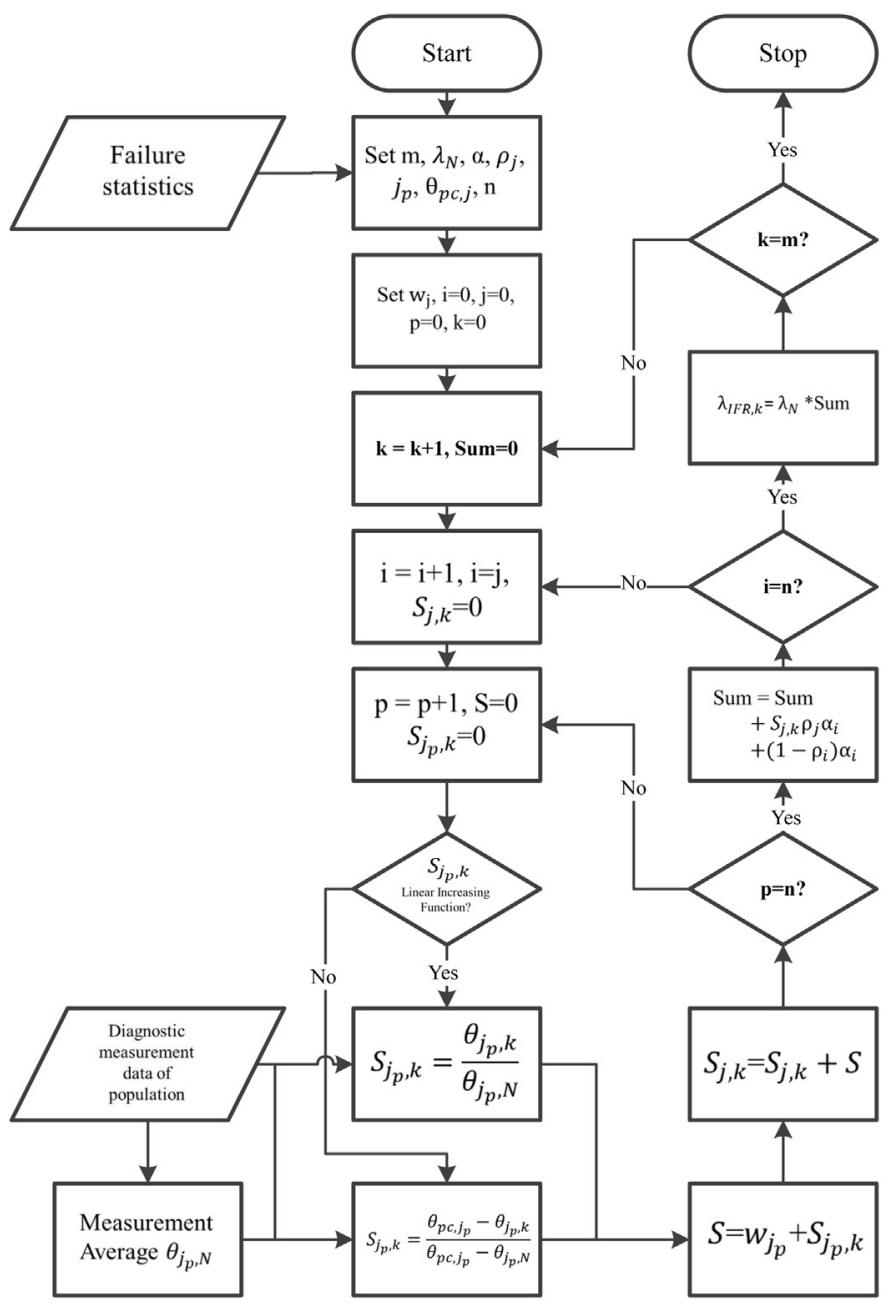

Fig. 3. Proposed method illustrated in graphical form as a flow chart.

all information is available to calculate the IFRs. For example, the calculation for transformer 1 is

$$
\begin{aligned}
\lambda_{I F R, 1}= & \underbrace{0.02}_{\lambda_{N}} *(\underbrace{1.91}_{S_{1,1}} * \underbrace{0.95}_{\rho_{1}} * \underbrace{0.32}_{\alpha_{1}}+(1-\underbrace{0.95}_{\rho_{1}}) * \underbrace{0.32}_{\alpha_{1}}) \\
& +0.02 * \sum_{i=2}^{8} \alpha_{i}=0.0256 .
\end{aligned}
$$

The computation of $\theta_{1, N}$ excludes obtained values that are above the critical limit. Thus, the average cannot represent a critical value in itself. The values $\theta_{1, N}$ and $\theta_{c l, 1}$, for each measurement, can be found in Table 1 . The critical limits were selected based on the IEEE guidelines [41,42]. The critical limit and the obtained diagnostic measures for the dissolved combustible gas (DCG) analysis are the summation of the DCG levels: $\mathrm{H}_{2}, \mathrm{CH}_{4}, \mathrm{C}_{2} \mathrm{H}_{2}, \mathrm{C}_{2} \mathrm{H}_{4}$, and $\mathrm{C}_{2} \mathrm{H}_{6}$.

The diagnostic measurement factors for measurements 1, 2, 4, 5, and 6 were calculated with the increasing linear function. This function was assumed due to the knowledge that when the diagnostic measures increase, the condition gets worse. The representation for $S_{j_{3}, k}$ for diagnostic measure $j_{3}$ is a decreasing linear function. The assumption was made that a perfect value equals $80 \mathrm{kV}$ to model the function. [42] recommends a test limit of $25 \mathrm{kV}$ (value for operation voltage class $\leq 69 \mathrm{kV}$ ) for the $1 \mathrm{~mm}$ gap for new oil in new equipment as acceptance level for decision-making. The value $80 \mathrm{kV}$ was determined by adjusting upward the highest obtained value in Table 1.

\section{Discussion of results}

The computed IFRs for all 30 transformers are presented as numerical values in Table 1 and graphically illustrated in a bar graph in Fig. 4c. Note that in Fig. 4a-c, the initial average failure rate is $\lambda_{N}=0.02$ which is the origin of computations for the IFRs. The results present higher failure rates for transformer 4, 11-15, 17, 19 , and 23. Particularly, transformer 19 with 0.1373 has the highest failure rate of the population. Analyzing the obtained diagnostic measures of transformer 19 indicates substantial negative deviation of the water content, dissipation factor, and 2-furfuraldehyde compared to the average of the population. Considering the IEEE guidelines [41,42] and further literature studies, especially, for the 2 -furfuraldehyde content $[5,43]$, we can conclude that the transformer is in a critical condition. In [29], power transformer less than 25 MVA with the 'worst' inspection outcome have a failure rate of 0.14 . Comparing the IFR 0.1373 of transformer 19 with the failure rate 0.14 , it shows that the results of the proposed method are accurate.

Likewise, this could also be conducted for the other aforementioned transformers with a considerably higher failure rate. These transformers also have high values of primarily 2-furfuraldehyde and can be classified as transformers being in a critical condition. A more detailed discussion of the population condition is available in [12].

Fig. $4 a-c$ depict the results when different confidence levels are used. Fig. 4c illustrates the case if the measurements have a stated confidence level of $95 \%$, there is an assurance of $95 \%$ that the stated $\mathrm{CI}$ contains the target value. Here, the IFRs are more detailed and it is possible to identify transformers that are in a better or worse condition compared to the initial average. In Fig. $4 \mathrm{~b}$, the probability is $68 \%$ that the stated $\mathrm{CI}$ contains the target value. The resulting limits are $\theta \pm \sigma$. In this case, it is less certain that the measurement was successful. This leads to results which are closer to the initial average failure rate. If the probability is determined to be $5 \%$ that the target value is within the stated $\mathrm{CI}$, the results are close to the initial average failure rate because the measurement process is not successful. A probability of $0 \%$ would mean that there is no additional information about the component and the average failure rate $\lambda_{N}$ is used. In general, the average failure rate is the starting point for component calculations and as more information from measurements is available, the more can be stated about the individual components. Comparing the cases in Fig. $4 a$, b, and c, also underlines the importance of reliable data and measurement quality. 


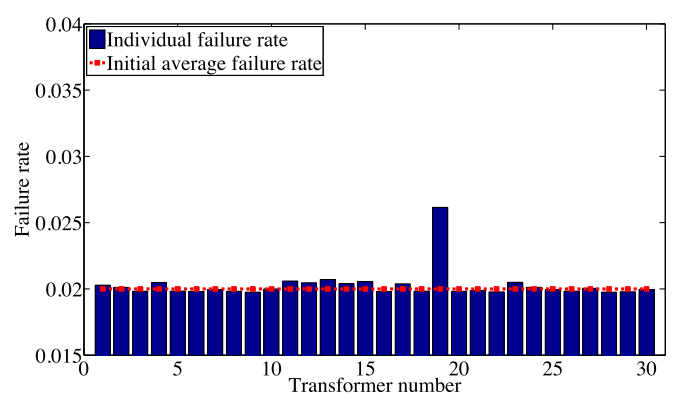

(a) Individual failure rates for transformers 1-30 with the initial average failure rate of 0.02 and a confidence interval of $5 \%$

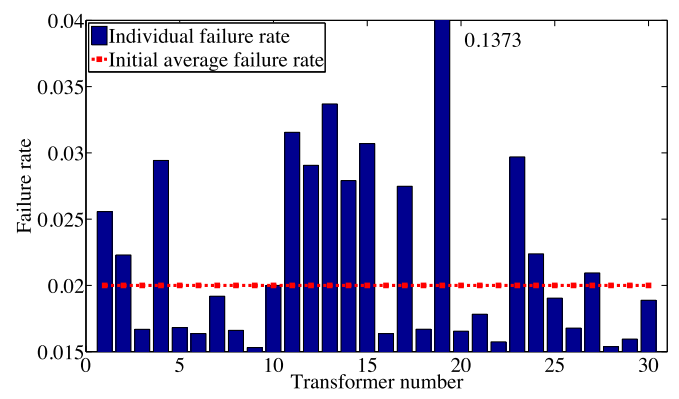

(c) Individual failure rates for transformers 1-30 with the initial average failure rate of 0.02 and a confidence interval of $95 \%$

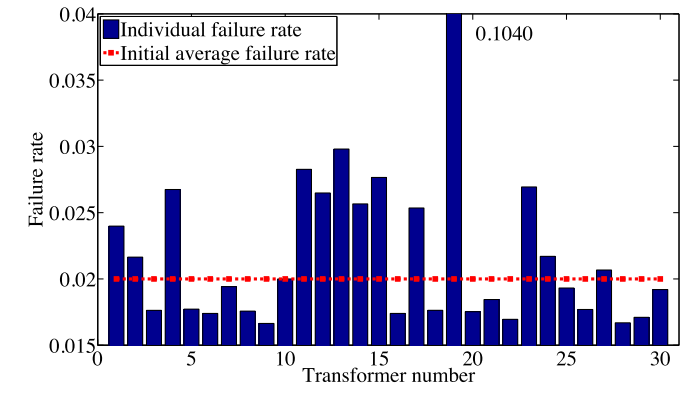

(b) Individual failure rates for transformers 1-30 with the initial average failure rate of 0.02 and a confidence interval of $68 \%$

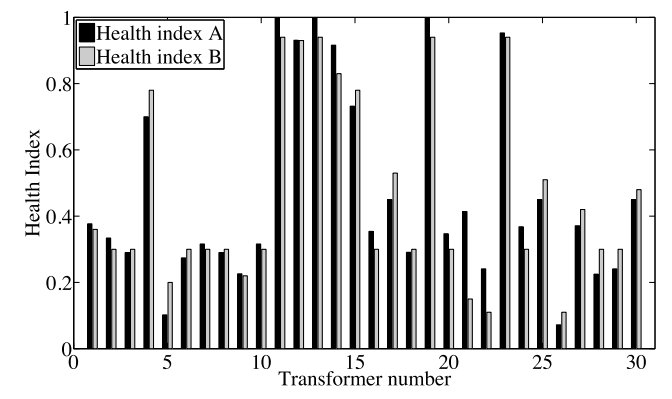

(d) Calculated health indices in [12] with health index A (weighting method) and health index B (fuzzy logic)

Fig. 4. Graphical illustration of the computed individual failure rates with different stated confidence intervals and the health indices A and B.

The estimation of the IFR is also valuable for system reliability calculations. The IFRs can be used in event tree analysis, reliability block diagrams, fault trees, etc.. This enables a more precise reliability evaluation on system level.

\section{Comparing the IFRs to health indices}

The proposed method estimates the probability of failure of the investigated transformer population rather than directly classifying in which condition certain transformers are. The categorization of a transformer condition requires in-depth knowledge about the diagnostic measurements to rank a transformer as good or bad. Nevertheless, the IFRs highlight the transformer with the highest failure rate, and thus the assumption is made that the computed results are reflecting the transformer condition. Therefore, the IFRs could be used for maintenance decision-making and compared to health indices.

In the previous section, the transformers $4,11-15,19$, and 23 were identified as transformers with a higher probability of failure. Table 1 also shows the computed health indices from [12] and how the values were graded by the authors. The health indices are computed with two different methods. Health index A was estimated by a Canadian consulting company which used a weighting approach. Health index B was calculated by using the fuzzy logic methodology. These two methods were compared and the results match with $96.7 \%$, according to [12]. Both methods classified transformers 4, $11-15,19$, and 23 as bad and recommended an immediate replacement. The comparison of the computed IFRs to the health indices in [12] shows that the results are plausible. The health indices are founded on expert knowledge or based on literature studies and are therefore a reliable benchmark.

The authors in [12] also state that transformers in a bad condition have a high risk of failure and that transformers in a very bad condition have an even higher risk of failure and, consequently, need replacement sooner. The proposed method estimates the probability of failure for a transformer. Examining Fig. 4d demonstrates the impact of different experts on the same transformer population. Despite similar results, the deviation is $64 \%$ of the health index values for transformer 21 and expressed as linguistic terms, it differs from very good to moderate. Both terms are computed on expert knowledge and a judgment which result is more correct is not possible.

Careful attention should be given to the interpretation of the health indices. The health indices are based on expert knowledge, hence, the method of calculation and the health index criteria and data should be clear to evaluate the results. Fig. 2 illustrates that $46 \%$ of failures are attributed to the tap changer and bushings of the transformer. Investigating the criteria of health index B shows that the failure locations for tap changer and bushings have not been included in the calculation. Therefore, the condition of the transformer becomes less valid because a transformer in a good condition can fail due to other reasons. This misleading interpretation is excluded in the method of the IFR since no condition classification is made. However, the IFRs reflect all defined failure locations and enable the opportunity to easily include condition information of diagnostic measures into the computation. Consequently, the IFRs will become more accurate. In addition, the method of IFR can be applied to all types of components. Only the input data of the average failure rate, the failure statistics, and the condition information are mandatory to conduct an analysis of the components.

A disadvantage of the proposed method, in terms of maintenance decision-making, is that a classification of the measured values is necessary to state how critical the condition of a single transformer is. For example, we identified transformer 19 with the highest failure rate but consulting IEEE guidelines or expert knowledge was required to state that the transformer needs replacement in the near future. 
Another drawback of the proposed method is the weighting of multiple measurements based on experience. This approach is used as a result of the often limited amount of historical measurement and failure data of power system equipment which must be correlated to failures to gain meaningful weights.

The set of functions for $S_{j, k}$ was limited by the assumption that there is a linear relationship between measurements and the failure rate. This limitation also influences the accuracy of the computed IFRs. An extended and improved set of functions would increase the accuracy of the IFRs which results in a better applicability as decision support. Furthermore, $S_{j, k}$ is based on the average of the obtained diagnostic measures. If the component population is in an overall bad condition, the method cannot specifically illustrate it. The factor $S_{j, k}$ describes the deviation from the average of the component population and thereby indicates differences of the probability of failure among the components. This is an essential characteristic of the proposed method.

An advantage of the proposed method, compared to the health indices, is the efficiency to calculate results if investigation of the transformer population is demanded. The computation is simple and can be executed without further knowledge. For example, health indices that use fuzzy logic or artificial neural networks need experience and knowledge about the techniques itself to gain reasonable results. Therefore, the presented technique can be implemented simply as an asset management tool into a utility without acquiring extra knowledge, software, and computation resources.

Software tools such as the Dynamic Transformer Management Program (DTMP) from ABB have been developed to manage large transformer fleets [16]. The DTMP also uses condition information and expert knowledge to calculate a "total risk of failure", compare individual asset details to the fleet, and provide recommendations for maintenance and replacement. This advanced asset management tool was mainly developed to handle large transformer fleets and "big data" from assets. From a utility viewpoint, when small transformer fleets need to be evaluated, the proposed method is able to present results without an asset management system. This could become even more significant if no advanced asset management system is implemented in a utility.

\section{Conclusion}

The demand for optimized transformer life-time has led to several condition monitoring methods with the attempt to analyze the transformer condition as precisely as possible. Health indices were developed to combine diagnostic data and expert knowledge to estimate a transformer condition for further asset management decision-making.

The presented methodology utilizes diagnostic measurements and failure statistics to quantify an individual failure rate to a transformer instead of using the average failure rate of the population. It was demonstrated that the suggested method computes accurate results and indicates transformers that have a higher probability of failure. The IFRs were also compared to two health indices and thereby the ability to use the results for maintenance decision-making was proven. Furthermore, the comprehensibility of the method makes it applicable for implementing it in electric utilities. Especially, when just small population sizes are given and results are urgent the method enables valid results with minimal complexity. Therefore, the method of IFRs becomes an option to an advanced asset management tool such as DTMP. Finally, the approach also underlines the importance of precise and accurate data and how it gives more confidence in decision-making.

\section{Future work}

The environmental impact on a component is important to consider when analyzing the condition. The proposed method partly includes the environmental impact through failure statistics and measurements which are related to it. Nevertheless, how to incorporate the environmental impact more specifically has to be further investigated. In a power system context, the environmental impact can be separated into statical and dynamical factors. For example, the wear of a component is influenced by the stress of the surroundings such as the weather. The weather is a dynamical factor because it is changing over time and thereby impacting the condition. Additional factors could be location, seasonal variations, or construction work. The impact of events which cause direct failures should also be considered in future studies. Therefore, future work should investigate the impact of static and dynamic factors on the failure rate.

In addition, the formalization of the method for time dependent measurement data is important. The failure rate over a time horizon could indicate trends which improve the method's ability for maintenance decision making.

Alternatively of using the failure locations as input vector for the method, the failure mode statistics can also be used. The allocation of diagnostic measurements towards failure modes might be more suitable in specific cases, especially, when certain functions of an equipment type are of greater interest rather than its subcomponents. However, this would have an impact on the results and thus should be investigated in subsequent work.

\section{Acknowledgement}

The authors would like to thank the Energiforsk $A B$ risk analysis program for funding the project.

\section{References}

[1] A.K. Jardine, D. Lin, D. Banjevic, A review on machinery diagnostics and prognostics implementing condition-based maintenance, Mech. Syst. Signal Process. 20 (7) (2006) 1483-1510, http://dx.doi.org/10.1016/j.ymssp.2005.09.012.

[2] W. Wang, Condition-based Maintenance Modelling, Springer Series in Reliability Engineering, Springer, London, 2008, http://dx.doi.org/10.1007/978-184800-011-7_5.

[3] M. Wang, A. Vandermaar, K. Srivastava, Review of condition assessment of power transformers in service, IEEE Electr. Insul. Mag. 18 (6) (2002) 12-25, http://dx.doi.org/10.1109/MEI.2002.1161455.

[4] J. Singh, Y. Sood, R. Jarial, Condition monitoring of power transformers - bibliography survey, IEEE Electr. Insul. Mag. 24 (3) (2008) 11-25, http://dx.doi.org/ 10.1109/MEI.2008.4591431.

[5] A.E. Abu-Elanien, M. Salama, Asset management techniques for transformers, Electr. Power Syst. Res. 80 (4)(2010) 456-464, http://dx.doi.org/10.1016/j.epsr. 2009.10.008.

[6] D. Hughes, G. Dennis, J. Walker, C. Williamson, Condition Based Risk Management (CBRM) - Enabling Asset Condition Information to be Central to Corporate Decision Making, Springer, London, 2006.

[7] D. Hughes, The use of "Health Indices" to determine end of life and estimate remnant life for distribution assets, in: Proceedings of the 17 th International Conference on Electricity Distribution (CIRED), 2003, pp. 5-15.

[8] A. Naderian, S. Cress, R. Piercy, F. Wang, J. Service, An approach to determine the health index of power transformers, in: Conference Record of the 2008 IEEE International Symposium on Electrical Insulation, 2008. ISEI 2008, 2008, pp. 192-196, http://dx.doi.org/10.1109/ELINSL.2008.4570308.

[9] T. Hjartarson, S. Otal, Predicting future asset condition based on current health index and maintenance level, in: IEEE 11th International Conference on Transmission Distribution Construction, Operation and Live-Line Maintenance, 2006. ESMO 2006, 2006, http://dx.doi.org/10.1109/TDCLLM.2006.340747.

[10] E. Figueroa, Managing an aging fleet of transformers, in: 6th Southern Africa Regional Conference, Cigre, 2009.

[11] A. Jahromi, R. Piercy, S. Cress, J. Service, W. Fan, An approach to power transformer asset management using health index, Electr. Insul. Mag. IEEE 25 (2) (2009) 20-34, http://dx.doi.org/10.1109/MEI.2009.4802595.

[12] A. Abu-Elanien, M. Salama, M. Ibrahim, Calculation of a health index for oil-immersed transformers rated under $69 \mathrm{kV}$ using fuzzy logic, IEEE Trans. Power Deliv. 27 (4) (2012) 2029-2036, http://dx.doi.org/10.1109/TPWRD. 2012.2205165. 
[13] M. Arshad, S. Islam, A novel fuzzy logic technique for power transformer asset management, in: Conference Record of the 2006 IEEE Industry Applications Conference, 2006. 41st IAS Annual Meeting, Vol. 1, 2006, pp. 276-286, http:// dx.doi.org/10.1109/IAS.2006.256536.

[14] A. Ashkezari, H. Ma, T. Saha, C. Ekanayake, Application of fuzzy support vector machine for determining the health index of the insulation system of in-service power transformers, IEEE Trans. Dielectr. Electr. Insul. 20 (3) (2013) 965-973. http://dx.doi.org/10.1109/TDEI.2013.6518966.

[15] J. Petersson, K. Persson, L. Walldén, N. Fantana, Condition based evaluation of net transformers-experience from a new ranking procedure, in: CIGRE Paris Conference Paper, 2002, pp. 12-108.

[16] L. Cheim, P. Lorin, L. Lin, P. Patel, J. Vines, A novel dynamic fleet wide condition assessment tool of power transformers, in: Cigre SC A2 and C4 Joint Colloquium, Zurich, Switzerland, 2013.

[17] A. Abu-Elanien, M. Salama, M. Ibrahim, Determination of transformer health condition using artificial neural networks, in: International Symposium on Innovations in Intelligent Systems and Applications (INISTA), 2011, pp. 1-5, http://dx.doi.org/10.1109/INISTA.2011.5946173.

[18] M.H. Bollen, Literature Search for Reliability Data of Components in Electric Distribution Networks, Eindhoven University of Technology, Faculty of Electrical Engineering, 1993.

[19] R.E. Brown, Electric Power Distribution Reliability, CRC Press, 2008.

[20] A. Moradkhani, M.R. Haghifam, M. Mohammadzadeh, Bayesian estimation of overhead lines failure rate in electrical distribution systems, Int. J. Electr. Power Energy Syst. 56 (2014) 220-227, http://dx.doi.org/10.1016/j.ijepes.2013.11. 022.

[21] L. Bertling, R. Allan, R. Eriksson, A reliability-centered asset maintenance method for assessing the impact of maintenance in power distribution systems, IEEE Trans. Power Syst. 20 (1) (2005) 75-82, http://dx.doi.org/10.1109/ TPWRS.2004.840433.

[22] P. Birkner, Field experience with a condition-based maintenance program of 20-kV XLPE distribution system using IRC-analysis, IEEE Trans. Power Deliv. 19 (1) (2004) 3-8, http://dx.doi.org/10.1109/TPWRD.2003.820421.

[23] F. Besnard, K. Fischer, L. Bertling, Reliability-centred asset maintenance - a step towards enhanced reliability, availability, and profitability of wind power plants, in: 2010 IEEE PES Innovative Smart Grid Technologies Conference Europe (ISGT Europe), 2010, pp. 1-8, http://dx.doi.org/10.1109/ISGTEUROPE. 2010.5638986 .

[24] K. Fischer, F. Besnard, L. Bertling Reliability-centered maintenance for wind turbines based on statistical analysis and practical experience, IEEE Trans. Energy Convers. 27 (1) (2012) 184-195, http://dx.doi.org/10.1109/TEC.2011. 2176129.

[25] F. Besnard, L. Bertling, An approach for condition-based maintenance optimization applied to wind turbine blades, IEEE Trans. Sustain. Energy 1 (2) (2010) 77-83, http://dx.doi.org/10.1109/TSTE.2010.2049452.

[26] Y. Wang, H. Pham, A multi-objective optimization of imperfect preventive maintenance policy for dependent competing risk systems with hidden failure, IEEE Trans. Reliab. 60 (4) (2011) 770-781, http://dx.doi.org/10.1109/TR. 2011.2167779.

[27] M. Rausand, A. Høyland, System Reliability Theory: Models, Statistical Methods, and Applications, vol. 396, John Wiley \& Sons, 2004.

[28] R. Billinton, R. Allan, Reliability Evaluation of Power Systems, Springer Science \& Business Media, 2013.

[29] R. Brown, Failure rate modeling using equipment inspection data, in: Power Engineering Society General Meeting. IEEE vol. 1, 2004, pp. 693-700.

[30] J. Bowles, Commentary - caution: constant failure-rate models may be hazardous to your design, IEEE Trans. Reliab. 51 (3) (2002) 375-377, http://dx.doi. org/10.1109/TR.2002.801850.

[31] J.L. Velasquez-Contreras, M.A. Sanz-Bobi, S.G. Arellano, General asset management model in the context of an electric utility: application to power transformers, Electr. Power Syst. Res. 81 (11) (2011) 2015-2037.

[32] J. Pathak, Y. Jiang, V. Honavar, J. McCalley, Condition data aggregation with application to failure rate calculation of power transformers, in: Proceedings of the 39th Annual Hawaii International Conference on System Sciences, HICSS '06., vol. 10, 2006, http://dx.doi.org/10.1109/HICSS.2006.93, pp. 241a.

[33] J. Pan, Z. Wang, D. Lubkeman, Condition based failure rate modeling for electric network components, in: Power Systems Conference and Exposition, 2009, PSCE '09. IEEE/PES, 2009, pp. 1-6, http://dx.doi.org/10.1109/PSCE.2009. 4839968.

[34] J. Yong, Condition-based hazard rate estimation and optimal maintenance scheduling for electrical transmission system, Retrospective Theses and Dissertations. Paper 1526.

[35] J. Rosenlind, P. Hilber, H. Tavakoli, Frequency response analysis (FRA) in the service of reliability analysis of power transformers, in: CMDM 2012, 2012.

[36] J.D. Kalbfleisch, R.L. Prentice, The Statistical Analysis of Failure Time Data, vol. 360, John Wiley \& Sons, 2011.

[37] S. Tenbohlen, F. Vahidi, P. Müller, J. Gebauer, M. Krüger, Zuverlässigkeitsbewertung von leistungstransformatoren, in: CIGRE Working Group A 2.37 Transformer Reliability Survey, 2012.

[38] JCGM, JCGM 104: 2009 Evaluation of Measurement Data - An Introduction to the "Guide to the Expression of Uncertainty in Measurement" and Related Documents, 2009

[39] JCGM, JCGM 100:2008 Evaluation of Measurement Data - Guide to the Expression of Uncertainty in Measurement, 2008.

[40] R. Willink, Measurement Uncertainty and Probability, Cambridge University Press, 2013.

[41] IEEE guide for the interpretation of gases generated in oil-immersed transformers, in: IEEE Std C57. 104-2008 (Revision of IEEE Std C57. 104-1991), 2009, pp. 1-36, http://dx.doi.org/10.1109/IEEESTD.2009.4776518.

[42] IEEE guide for acceptance and maintenance of insulating oil in equipment, in: IEEE Std C57. 106-2006 (Revision of IEEE Std C57. 106-2002), 2006, pp. 1-36, http://dx.doi.org/10.1109/IEEESTD.2006.371041.

[43] A. Emsley, X. Xiao, R. Heywood, M. Ali, Degradation of cellulosic insulation in power transformers. part 3: Effects of oxygen and water on ageing in oil, IEE Proceedings-Science, Measurement and Technology 147 (3) (2000) 115-119.

[44] A. Bossi, J. Dind, J. Frisson, U. Khoudiakov, An international survey of failures in large power transformers in service, in: Final Report of the CIGRE Working Group 12.05, Electra (88), 1983, pp. 21-47.

[45] V. Sokolov, S. Tsurpal, A. Drobyshevski, Reliability problems with large power transformers and shunt reactors. Typical failure modes and failure causes, in: CIGRE A2 COLLOQUIUM, 2005.

[46] R. Jongen, P. Morshuis, J. Smit, A. Janssen, E. Gulski, A statistical approach to processing power transformer failure data, in: Cired 19th International Conference on Electricity Distribution, 2007

[47] J. Jagers, J. Khosa, P. De Klerk, C. Gaunt, Transformer reliability and condition assessment in a south African utility, in: International Symposium on High Voltage Engineering, Ljubljana, 2007.

[48] A. Franzén, L. Bertling, State of the art-life time modeling and management of transformers, KTH - Royal Institute of Technology, Stockholm.

[49] M. Lessard, et al., Furanic compounds for diagnosis, in: CIGRE Working Group D1.01 (TF 13), 2012

[50] Standard test method for water in insulating liquids by coulometric karl fischer titration, ASTM Standard D1533 - 12. doi:10.1520/D1533-12.

[51] Standard test method for acid number of petroleum products by potentiometric titration, ASTM Standard D664 - 11a doi:10.1520/D0664-11A

[52] Standard test method for dielectric breakdown voltage of insulating liquids using vde electrodes, ASTM Standard D1816 - 12 doi:10.1520/D1816-12.

[53] Standard test method for dissipation factor (or power factor) and relative permittivity (dielectric constant) of electrical insulating liquids, ASTM Standard D924 - 08 doi:10.1520/D0924-08.

[54] Standard test method for analysis of gases dissolved in electrical insulating oil by gas chromatography, ASTM Standard D3612 - 02 (Reapproved 2009) doi:10.1520/D3612-02R09.

[55] Standard test method for furanic compounds in electrical insulating liquids by high-performance liquid chromatography (HPLC), ASTM Standard D5837 - 12 doi:10.1520/D5837-12. 